\title{
Are Bone Allografts Safe and Effective for Today's Dental Practitioner?
}

\author{
Brian Samsell ${ }^{1}$, Mark Moore ${ }^{1}$, Giampietro Bertasi ${ }^{2}$, Sergio Spinato ${ }^{3}$, Fabio Bernardello ${ }^{4}$, Alberto Rebaudi ${ }^{5}$, Gian Luca Sfasciotti ${ }^{6}$ and Ralph Powers ${ }^{1^{\star}}$ \\ ${ }^{1}$ Institute of Regenerative Medicine, LifeNet Health, 1864 Concert Drive, Virginia Beach, VA 23453, USA \\ ${ }^{2}$ University of Padua, Padua, Italy \\ ${ }^{3}$ Department of Biomedical and Neuromotor Sciences, School of Dentistry, Unit of Periodontology and Implantology, University of Bologna, Bologna, Italy \\ ${ }^{4}$ Private Practice, Terranegra di Legnago, Verona, Italy \\ ${ }^{5}$ Private Practice, Genoa, Italy \\ ${ }^{6}$ Department of Dentistry and Maxillo-facial Sciences, Sapienza University of Rome, Rome, Italy
}

${ }^{*}$ Corresponding author: Ralph Powers, DDS, Institute of Regenerative Medicine, LifeNet Health, 1864 Concert Drive, Virginia Beach, VA 23453, USA, Tel: 1-757-609-4533; E-mail: ralph_powers@lifenethealth.org

Rec Date: July 09, 2014; Acc Date: Oct 27, 2014; Pub Date: Nov 3, 2014

Copyright: (C) 2014 Samsell B et al. This is an open-access article distributed under the terms of the Creative Commons Attribution License, which permits unrestricted use, distribution, and reproduction in any medium, provided the original author and source are credited.

\section{Abstract}

A wide variety of dental procedures, including ridge and sinus augmentation, treatment of bony defects, and extraction socket preservation, may require a bone grafting material. To meet this need, there are many choices available including alloplasts, xenografts, autografts, and allografts. In particular, allografts, being a natural, human biological matrix and readily available have proven clinically reliable. However, not all allografts are equal in terms of processing, sterility, and proven clinical performance for dental applications. Here, we review the use of disinfected and terminally sterilized bone allografts for dental applications.

Keywords: Allograft; Defect; Preservation; Implant; Augmentation; Bone regeneration; OraGraft

\section{Introduction}

Many dental procedures, including ridge augmentation, implant placement, or intrabony periodontal defect treatment, require the growth of new bone to be successful. Growth of new bone has become a critical element for procedures performed by the modern dental specialist. New bone growth is facilitated by osteogenesis, osteoinduction, or osteoconduction [1]. Osteogenesis utilizes osteoblasts to generate new bone. Osteoinduction involves signaling molecules or growth factors, inducing local cells into osteoblastic or odontoblastic activity. Osteoconduction is a process whereby a graft acts as a scaffold for new bone formation, but requires the presence of bone forming cells, typically from the host. When using common bone void fillers, it is important to be aware of the method of bone growth when comparing the four major graft types: alloplastic, xenogeneic, autogenous, and allogeneic grafts. Alloplasts, commonly made from hydroxyapatite (HA)or $\beta$-tricalcium phosphate $(\beta-\mathrm{TCP})$, provide an osteoconductive scaffold. They lack both osteogenenic and osteoinductive properties, so they are often supplemented with autograft bone, bone marrow aspirate (BMA), or bone morphogenetic proteins (BMP) to provide better functionality [2].

Xenografts for human implantation are typically derived from bovine, porcine, equine or coralline apatite matrices. In a similar way to alloplastic grafts, xenografts may experience immunogenic reactions to the foreign substance and complications can occur [3]. In addition, xenografts often require aggressive treatment with cytotoxic chemicals, such glutaraldehyde and formaldehyde, in order to prevent immunogenic reactions [4]. These treatments can affect mechanical properties and behavior [4,5]. Finally, when using xenografts, surgeons need to be aware of religious or ethical considerations of their patients.
Autogenous grafts are taken from a donor site within the patient, often from an intra-oral site such as the ramus of the mandible, the chin, or the tuber maxillae or sometimes from an extra-oral site as the hip, tibia, rib, or calvarium for larger grafts. Favorably, autografts possess osteogenic properties and no immunogenic issues [1]; however, there are disadvantages. There can be patient pain and morbidity at the harvest site [6-10] as well as insufficient usable graft material procured due to donor site atrophy or an underestimate of availability. The associated costs and time involved with donor site surgery and resultant complications may be significant. The surgeon must spend more surgical time by first harvesting the autograft and then trimming it into the correct size for implantation, in addition to risk of donor site infection [11-13].

Allografts come from tissue recovered from qualified deceased human donors. Allograft bone used in dental applications may be demineralized, which exposes osteoinductive growth factors, thus promoting new bone formation [14]. In addition, the natural osteoconductive property of human bone facilitates the generation of more new bone formation and cellular proliferation than either xenografts or alloplasts alone [15]. Furthermore, the use of an allograft enables the surgeon to avoid the immunogenic reaction possible with alloplasts and xenografts, and also the donor site morbidity and associated complications when using autografts. These advantages position allografts as a favorable option for both surgeon and patient, although variability exists in allograft processing and sterility. Here, we review sterility considerations in choosing an allograft and report on clinical evidence using a particular manufactured bone allograft for dental applications.

\section{Special note on allograft sterility}

The possibility of disease transmission is an often cited risk of allograft use. Tissue banks accredited through the American 
Page 2 of 6

Association of Tissue Banks (AATB) have essentially negated this risk through stringent donor screening, recovery, and disinfection processes. However, some tissue banks, accredited or non-accredited, while using aseptic recovery and processing techniques, do not offer the additional safety margin of terminal sterilization. Allografts that are minimally processed, and then distributed frozen are at risk of transmitting viruses such as HIV and HCV [16]. To optimally eliminate bacterial agents and inactivate viruses, aseptic recovery and stringent disinfection procedures can be used in conjunction with a validated terminal sterilization technique.

Sterility is measured using a Sterility Assurance Level (SAL) as a measure of the probability of the presence of a viable microorganism. For example, a $10^{-3} \mathrm{SAL}$ would mean no more than 1 in 1000 grafts would contain a viable microorganism. This $10^{-3} \mathrm{SAL}$ corresponds to that assured by validated, aseptic transferring steps of already sterile product and is recommended by Food and Drug Administration (FDA) only for medical devices that do not cross the skin barrier [17]. In contrast, a $10^{-6} \mathrm{SAL}$ indicates no more than 1 in 1,000,000 grafts are would contain a single viable microorganism. A SAL of at least $10^{-6}$ is considered sterile when achieved only when using a validated process [18] and Centers for Disease Control and Prevention (CDC) Guidelines assert implanted medical devices should be sterilized [19] to this level. This level of terminal sterilization can be achieved with alloplasts, xenografts, and allografts using gamma irradiation. A high level of sterility is expected in surgical settings for many operating room supplies, especially medical implants. However, some allografts do not achieve this level of sterility. While older studies have reported that high doses of gamma irradiation can damage tissue [20,21], proper irradiation conditions to ensure material sterility without impacting clinical performance take into account the following four criteria: target dose, dose range, temperature during irradiation, and tissue treatment prior to irradiation [22]. Keeping the graft at low temperature during irradiation minimizes the creation of free radicals that can negatively affect tissue properties [23] and should be considered a critical step. Under controlled conditions, terminal sterilization and viral inactivation can be achieved without negative impact on biomechanical properties or clinical performance of allograft tissues [20,24,25].

Thus, while many tissue banks distribute allografts for dental procedures, variability in disinfection and sterilization methods leads the clinician to consult published literature in chosing an allograft.

\section{Properties of disinfected, terminally sterilized bone allograft for dental applications}

This review will focus on highly disinfected, terminally sterilized bone allografts. In particular, OraGraft ${ }^{\bullet}$ (LifeNet Health, Virginia Beach, VA), hereafter referred to as OG bone allograft, is currently processed using a proprietary and patented method that removes greater than $99 \%$ of bone marrow and blood elements from the internal blood matrix [26]. This process utilizes detergents, isopropanol, and hydrogen peroxide to clean and disinfect the bone. In addition, the graft is subjected to a terminal sterilization process utilizing less than $2 \mathrm{Mrad}$ of gamma irradiation at dry ice temperatures to render OG bone allografts sterile to a SAL of $10^{-6}$ [27]. It should be noted that this low dosage irradiation is sufficient to sterilize bone material if the manufacturer practices stringent processes related to the use of tissues deriving from only medically suitable and qualified donors and recovered in an aseptic environment using zone recovery techniques and then utilizing a battery of tests to detect bacteria (both aerobic and anaerobic), fungi, viruses and infectious diseases [28,29] including the use of Nucleic Acid Tests (NAT). NAT testing provides a 12 day window for HIV-1 on each donor instead of the longer 22-day window with traditional antibody tests [30]. In addition to testing, subsequent cleaning and disinfection further reduces the risk of disease transmission by removing blood and bone marrow. The terminal sterilization process is validated to ensure the microbial SAL of $10^{-6}$ and low dosage irradiation has demonstrated viral inactivation [25] (Table 1).

\begin{tabular}{|c|c|c|c|c|c|}
\hline & Aseptic Processing & Eto & Gamma Irradiation & $\begin{array}{l}\text { Chemical } \\
\text { Sterilants }\end{array}$ & $\begin{array}{l}\text { Sterilization } \\
\text { used for } \text { Process } \\
\text { Allograft }\end{array}$ \\
\hline Kills bacteria & No & 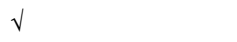 & $\sqrt{ }$ & $\sqrt{ }$ & 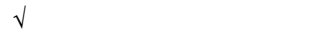 \\
\hline Kills fungi & No & $\sqrt{ }$ & $\sqrt{ }$ & $\sqrt{ }$ & $\sqrt{ }$ \\
\hline Kills spores & No & $\sqrt{ }$ & $\sqrt{ }$ & No & $\sqrt{ }$ \\
\hline Kills viruses & No & No & $\checkmark$ (dose-dependent) & No & $\sqrt{ }$ \\
\hline Removes blood and lipids & Surface Only & No & No & Surface Only & $\sqrt{ }$ \\
\hline Preserves strength & $\sqrt{ }$ & $\sqrt{ }$ & Decreases(dose-dependent) & $\sqrt{ }$ & $\mathrm{v}$ \\
\hline Preserves biocompatibility & $\sqrt{ }$ & $\sqrt{ }$ (dose-dependent) & $\sqrt{ }$ & $\sqrt{ }$ & $\sqrt{ }$ \\
\hline
\end{tabular}

Table 1: Comparison of different allograft processing methods $[29,30]$

Regarding potential impact of irradiation on important allograft properties, numerous studies have found no significant difference in the biomechanical properties [31-36] or clinical outcomes [37-39] between non-irradiated allografts and properly treated irradiated allografts. Additional studies have shown that irradiated demineralized bone matrices (DBM) retain comparable levels of osteoinductivity to DBMs that were not irradiated. Of particular interest, Diziedic-
Goclawska et al. [40] found DBM samples that were irradiated at room temperature were reabsorbed within five weeks while samples irradiated on dry ice had comparable results to non-irradiated samples. Wientroub and Reddi [41] noted similar osteoinductive properties of DBM between non-irradiated rat bone allografts and bone allografts irradiated at low temperatures. 
Many clinicians prefer the use of a demineralized bone matrix especially if desiring a more osteoinductive material. A bone graft can be labeled demineralized if it meets the American Association of Tissue Banks (AATB) standard definition of containing no greater than than $8 \%$ residual calcium. However, several reports indicate the optimal residual calcium range is approximately $1 \%-4 \%$ [42-45]. Bone containing residual calcium levels of $>4 \%$ are considered underdemineralized since osteoinductive growth factors remain trapped in the matrix, muting their clinical impact. Conversely, bone containing residual calcium levels of $<1 \%$ may be considered over-demineralized since osteoinductive growth factors may be either denatured by overexposure to the acidic demineralization solution or physically removed from the matrix in the solution, both conditions leading to diminished osteoinductive potential. To prepare demineralized bone matrix, OG bone allografts undergo a patented [46] technology to target an optimal calcium residual level of $1 \%-4 \%$. This method utilizes controlled pulses of acid solution to carefully demineralize bone to target levels without excessive acid exposure.

\section{Clinical studies using OG bone allografts}

While achieving an adequate degree of safety through advanced processing techniques is essential, clinical efficacy must be demonstrated. The following clinical studies all utilized OG bone allografts, including those grafts terminally sterilized since 2004. Aichelmann-Reidy et al. [47] used OG demineralized freeze-dried bone allograft (DFDBA) in a 20 patient, randomized study comparing calcium sulfate (CS) and polytetraflouroeythlyene. After 6 months follow-up, the authors reported "achiev[ing] 50\% or better resolution in both in 95\% $(18 / 19)$ of the sites" in both treatment groups and concluded with support for using CS combined with DFDBA for treating intrabony defects. Callan [48] used both DFDBA and freezedried fascia lata femoris to, respectively, fill and protect an osseous defect in a case series. The author recommended both allograft types after finding this technique "provide[d] an increased amount of alveolar bone for better implant placement." In a randomized, singlemasked study involving 40 patients, Gurinsky et al. [49] compared enamel derived matrix (ECM) only and ECM combined with DFDBA to treat intrabony periodontal defects. They concluded both treatments significantly improved the defects with the combined DFDBA treatment "yield[ing] statistically significant improvements in bone fill, crestal resorption, and percentage of site gaining greater than $50 \%$ and $90 \%$ bone fill when compared to ECM alone." Landi and Sabatucci [50] published a technique report which described utilizing DFDBA to successfully treat defects in the mandibular ridge to prepare the location for implantation. The authors note "keratinized tissue was fully recognizable around all of the implants" at six weeks postoperative. Wood and Mealey [51] also compared the efficacy of demineralization in a randomized, comparative study involving 40 patients implanted with either DFDBA or freeze dried bone allografts (FDBA). After 19 weeks follow-up, biopsies showed patients implanted with DFDBA had significantly greater amounts of new vital bone formation $(38.42 \%)$ and a lower mean percentage of residual grafts particles $(8.88 \%)$ than patients in the FDBA group $(24.63 \%$ and $25.42 \%$, respectively). Numerous other clinical studies have reported using processed DFDBA for repairing periodontal intrabony defects, inducing bone regeneration around implants, and maxillary sinus augmentation [52-56].

Several more clinical studies have been published that used nondemineralized OG FDBA for similar treatments [57-66]. In a prospective study comparing allograft only with allograft and autograft combination treatment, Beitlitum et al. [62] used FDBA to augment the alveolar ridge deficiencies of 50 patients. The authors found that not only did the FDBA alone yield good clinical results but concluded "there was no added clinical effect of the application of a layer of autogenous bone," indicating the autograft treatment was essentially equivalent to the allograft treatment alone. Kassolis et al. [58] concluded their study of 15 patients with support for using FDBA for maxillary sinus grafting noting that "FDBA in combination with PRP [platelet-rich plasma] provides a viable therapeutic alternative for implant site preparation." In a study designed to test for donorspecific HLA antibodies, Quattlebaum et al. [57] used FDBA to treat periodontal osseous defects in 20 patients. The authors were unable to detect any antibodies at intervals over a 3 month time period. Schwartz et al. [59] reported "excellent bone fill of the osseous defect" at six month follow-up in a case report where FDBA was mixed with an enamel matrix derivative to fill a palatal bony defect located on the maxillary incisor. In a recent case series, Spinato and Galindo-Moreno [66] saw "good preservation of soft and hard tissue architecture" after a one year follow-up in eight patients who were treated with a mixture of FDBA and DFDBA to prepare the maxillary extraction site for implant placement. Vidal et al. [63] reported a $100 \%$ success rate of implant placement (defined as grade 3 or $>1 \mathrm{~mm}$ of bone loss) after one year follow-up in a study consisting of 51 patients who had immediate implant placements. While the total number of patients who received FDBA was not specified, FDBA along with a collagen membrane was grafted onto sockets that had $>1 \mathrm{~mm}$ distance to the implant surface.

OG cancellous blocks are often used for maxillary and alveolar ridge augmentations [67-73]. Lyford et al. [67] used cancellous blocks to augment the alveolar ridge in a case series of 3 patients. The authors believe their work is the first published study of such treatment and concluded with support for allograft use stating "the cancellous block allograft may provide one such alternative treatment that meets the clinical requirements while satisfying the patient's expectations [reduced extent, time, costs of the surgical procedure]." Nissan et al. [68] published a study where they augmented deficient alveolar ridges for single-tooth implants in 9 patients with cancellous blocks. By 18 month follow-up, there was no bone loss below the first thread and all implants were still functional and in good condition. The authors noted that this technique has the advantages of "minimizing postoperative morbidity; elimination of second-stage implant placement surgery, reduced surgical trauma; minimal use of a provisional removable restoration; and the ability to satisfy esthetic demands in the shortest time possible." In 2011, Nissan et al. [69] published another similar, but larger study where they used 46 cancellous blocks to treat alveolar ridge deficiencies in 31 patients who required implants. They found $95.6 \%$ graft success (two failed bone grafts) and $98 \%$ immediate implant success with the single failed implant due to an automobile accident. The implant was reinserted and all implants had a $100 \%$ success rate (defined as clinical osseointegration) after a mean 34 month follow-up. Chaushu et al. [70] used cancellous blocks for maxilla sinus floor augmentation along with simultaneous implant placement for 28 patients. After a 27 months follow-up, the authors found a $94.9 \%$ success rate (defined as clinical osseointegration), although all implants considered failed were reinserted and osseointegrated. The histologic analysis revealed new bone formation "containing viable osteocytes merged with residual grafted bone." In a case study of a single patient with a 21 month follow-up, Wallace and Gellin [71] used cancellous blocks to augment the maxillary ridge for implant placement. Not only did the authors find the graft successful 
(allowed implant placement) but they suggested "cancellous block allografts could be a promising alternative to autogenous block grafts." Wallace and Gellin [72] reached the same conclusion when they followed this initial study up with a published 12 patient case series in 2010 that also had a 100\% graft success rate (no grafts were lost by 5 months following placement). The authors also had a $100 \%$ implant success rate as "no implants failed during the 4 month integration period." Wallace [73] recently published a study utilizing cancellous particulate FDBA in combination with a membrane composed of decellularized dermis to preserve extraction sites for implant placement in six patients. Histologic analysis showed a "significant percentage [average 12.8\%] of new bone regeneration after 12 weeks in molar extraction sites."

Very recently Bernardello et al. [74] performed the first human histologic evaluation of a two-stage crestal sinus elevation technique, utilizing $\beta$-TCP as radiographic tracer and mineralized human bone allograft as grafting material, in a severely atrophic maxilla. After a six month healing period, a core biopsy was taken and the histology highlighted a wide mineralized composite network of allograft granules connected by newly formed bone and osteoblast activities [75]. However, $\beta$-TCP resulted in poor contact with bone. The histologic outcomes of this report demonstrated significantly better behavior outcomes of FDBA than $\beta$-TCP.

\section{Conclusion}

While many different graft types are available for dental procedures, there are numerous advantages of allografts, as described here. However, allograft processing varies by manufacturer, and resulting product intended for dental applications may differ in sterilization assurance, osteoinductive potential, and proven clinical performance. One allograft option, referred to here as "OG bone allograft", is provided sterile to a SAL of $10^{-6}$ and with an extensive history of published studies to support clinical efficacy, makes this type of graft a valid option for the dental practitioner to consider.

\section{References}

1. Dimitriou R, Jones E, McGonagle D, Giannoudis PV (2011) Bone regeneration: current concepts and future directions. BMC Med 9: 66.

2. Giannoudis P, Dinopoulos H, Tsiridis E (2005) Bone substitutes: an update. Injury 36: S20-27

3. Miller L, Block J (2011) Perspectives on the clinical utility of allografts for bone regeneration within osseous defects: a narrative review. Orthop Res Rev 3: 31-37

4. Chandrasekaran R, Balasundari R, Sivasubramanian V, Cherian KM, Nayak V, et al. (2007) Cytotoxicity and sensitization studies of processed porcine xenografts. Ind J Thorac Cardiovasc Surg 23: 426-250

5. Jayakrishnan A, Jameela SR (1996) Glutaraldehyde as a fixative in bioprostheses and drug delivery matrices. Biomaterials 17: 471-484

6. Dublin N, Stewart L (2004) Oral complications after buccal mucosal graft harvest for urethroplasty. BJU Int 94: 867-869

7. Wood D, Allen S, Greenwell T, Mundy A (2004) The morbidity of buccal mucosal graft harvest for urethroplasty and the effect of nonclosure of the graft harvest site on postoperative pain. J Urol 172: 580-583

8. Fabbroni G, Loukota R, Eardley I (2005) Buccal mucosal grafts for urethroplasty: surgical technique and morbidity. Br J Oral Maxillofac Surg 43: 320-323

9. Sinha RJ, Singh V, Sankhwar SN, Dalela D (2009) Donor site morbidity in oral mucosa graft urethroplasty: implications of tobacco consumption. BMC Urol 9: 15.
10. Marolt D, Knezevic M, Novakovic G (2010) Bone tissue engineering with human stem cells. Stem Cell Res Ther 1: 10

11. Dahlin C, Johansson A (2011) Iliac crest autogenous bone graft versus alloplastic graft and guided bone regeneration in the reconstruction of atrophic maxillae: a 5-year retrospective study on cost-effectiveness and clinical outcome. Clin Implant Dent Relat Res 13: 305-310

12. Guided bone regeneration in the reconstruction of atrophic maxillae: a 5year retrospective study on cost-effectiveness and clinical outcome. Clin Implant Dent Relat Res 13: 305-10. http://www.ncbi.nlm.nih.gov/ pubmed/21087398

13. Cole D, Ginn T, Chen G, Smith B, Curl W, et al. (2005) Cost comparison of anterior cruciate ligament reconstruction: autograft versus allograft. Arthroscopy 21:786-790

14. Truedsson A, Hjalte K, Sunzel B, Warfvinge G (2013) Maxillary sinus augmentation with iliac autograft - a health-economic analysis. Clin Oral Implants Res 24: 1088-1093.

15. Wei L, Miron RJ, Shi B, Zhang Y (2013) Osteoinductive and osteopromotive variability among different demineralized bone allografts. Clin Implant Dent Relat Res: Epub ahead of print

16. Srouji S, Ben-David D, Funari A, Riminucci M, Bianco P (2012) Evaluation of the osteoconductive potential of bone substitutes embedded with schneiderian membrane- or maxillary bone marrowderived osteoprogenitor cells. Clin Oral Impl 24: 1288-1294

17. Salvucci J (2011) Bone tissue, lyophilized and stored at room temperature for 15 days or more, is not capable of transmitting HIV, HCV or HBV. Cell Tissue Bank 12: 99-104

18. Food and Drug Administration (2008) Submission and Review of Sterility Information in Premarket Notification (510(k)) Submissions for Devices Labeled as Sterile

19. ANSI/AAMI ST67: 2003/(R). Sterilization of health care productsrequirements for products labeled "STERILE"

20. Rutala W, Weber D (2008) The Healthcare Infection Control Practices Advisory Committee (HCPAC). Guideline for disinfection and sterilization in healthcare facilities. Centers for Disease Control and Prevention (CDC) Publication

21. Anderson MJ, Keyak JH, Skinner HB (1992) Compressive mechanical properties of human cancellous bone after gamma irradiation. J Bone Joint Surg Am 74: 747-752

22. Mitchell EJ, Stawarz AM, Kayacan R, Rimnac CM (2004) The effect of gamma irradiation on the fatigue crack propogation resistance of human cortical bone. J Bone Surg Am 86-A: 2648-2657

23. Samsell B, Moore M (2012) Use of controlled low dose gamma irradiation to sterilize allograft tendons for ACL reconstruction: biomechanical and clinical perspective. Cell Tissue Bank 13: 217-223

24. Hamer AJ, Stockley I, Elson RA (1999) Changes in allograft bone irradiated at different temperatures. J Bone Joint Surg Br 81:342-344

25. Block J (2006) The impact of irradiation on the microbial safety, biomechanical properties and clinical performance of musculoskeletal allografts. Orthopedics 29: 991-996

26. Moore M (2012) Inactivation of enveloped and non-enveloped viruses on seeded human tissues by gamma irradiation. Cell Tissue Bank 13: 401-407

27. US Patents $7,338,757 ; 6,743,574 ; 6,734,018$

28. Moore M, Linthurst Jones A, Gaskins B (2004) Adaptation of ANSI/ AAMI/ISO 11137 method 2B sterilization validation for medical devices to tissue banking. Presented American Association of Tissue Banks Annual Meeting. Chicago, IL

29. Vangsness CT (2004) Overview of allograft soft tissue processing. AAOS Bulletin

30. Micarelli A, Moore M, Peycelon F. Variation in allograft sterility levels: understanding the clinical and economic implications. White Paper

31. McGuire D, Hendricks S (2009) Allograft tissue in ACL reconstruction. Sports Med Arthrosc 17: 224-233

32. Gibbons M, Butler D, Grood E, Bylski-Austrow D, Levy M, et al. (1991) Effects of gamma irradiation on the initial mechanical and material 
properties of goat bone-patellar tendon-bone allografts. J Orthop Res 9 209-218

33. Goertzen M, Clahsen H, Burrig K, Schulitz K (1995) Sterilization of canine anterior cruciate allografts by gamma irradiation in argon. J Bone Joint Surg Br 77: 205-212

34. McGilvray K, Santoni B, Turner A, Bogdansky S, Wheeler D, et al. (2011) Effects of irradiation dose on the initial structural biomechanical properties properties of ovine bone-patellar tendon- bone allografts. Cell Tissue Bank 12: 89-98

35. Roche C, Gaskins B, Kuhn C, Moore M (2005) The effects of gamma irradiation on the biomechanical properties of soft tissue allografts. 29th Annual Meeting of the American Association of Tissue Banks. Hollywood, FL

36. Balsly C, Cotter A, Williams L, Gaskins B, Moore M, et al. (2008) Effect of low dose and moderate dose gamma irradiation on the mechanical properties of bone and soft tissue allografts. Cell Tissue Bank 9: 289-298

37. Greaves L, Hecker A, Brown C (2008) The effect of donor age and lowdose gamma irradiation on the initial biomechanical properties of human tibialis tendon allografts. Am J Sport Med 36: 1358-1366

38. Fanelli G, Giannotti B, Edson C (1996) Arthroscopically assisted combined anterior and posterior cruciate ligament reconstruction. Arthroscopy 12: 5-14

39. Sekiya J, Hussein E, Harner C (2002) Meniscal transplant combined with anterior cruciate ligament reconstruction. Oper Techn Sport Med 10: 157-164

40. Rihn J, Irrgang J, Chhabra A, Fu F, Harner C (2006) Does irradiation affect the clinical outcome of patellar tendon allograft ACL reconstruction. Knee Surg Sport Tra A 14: 885-896

41. Dziedzic-Goclawska A, Ostrowski K, Stachowicz W, Michalik J, Grzesik W (1991) Effect of radiation sterilization on the osteoinductive properties and the rate of remodeling of bone implants preserved by lypohilization and deep-freezing. Clin Orthop Relat Res 272: 30-37

42. Wientroub S, Reddi AH (1988) Influence of irradiation on the osteoinductive potential of demineralized bone matrix. Calcif Tissue Int 42: $255-260$

43. Zhang M, Powers RM, Wolfinbarger L (1997) Effect(s) of the demineralization process on the osteoinductivity of dermineralized bone matrix. J Periodontal 68: 1085-1092

44. Herold RW, Pashley DH, Cuening MF, et al. (1999) The effects of varying degrees of allograft decalcification on cultured porcine osteoclast cells. J Periodontol 73: 213-219

45. Mott D, Mailhot J, Cuenin M, Sharawy M, Borke J (2002) Enhancement of osteoblast proliferation in vitro by selective enrichment of demineralized freeze-dried bone allograft with specific growth factors. J Oral Implantol 28: 57-66

46. Turonis JW, McPherson JC 3rd, Cuening MF, Hokett SD, Peacock ME, et al. (2006) The effect of residual calcium in decalcified free-dried bone allograft in a critical-size defect in the Rattus norvegicus calvarium. J Periodontal 32: 55-62

47. US Patents 6,189,537; 6,534,095

48. Aichelmann-Reidy M, Heath C, Reynolds M (2004) Clinical evalutation of calcium in combination with demineralized freeze-dried bone allograft for the treatment of human intraosseous defects. J Periodontol 75: 340-347

49. Callan D (1993) Guided tissue regeneration without a stage 2 surgical procedure. Int J Periodontics Restorative Dent 13: 173-179

50. Gurinsky B, Mills M, Mellonig J (2004) Clinical evaluation of demineralized freeze-dried bone allograft and enamel matrix derivative versus enamel matrix derivative alone for the treatment of periodontal osseous defects in humans. J Periodontol 75: 1309-1318

51. Landi L, Sabatucci D (2001) Plastic surgery at the time of membrane removal around mandibular endosseous implants: a modified technique for implant uncovering. Int J Periodontics Restorative Dent 21: 1-7
52. Wood RA, Mealey BL (2012) Histologic comparison of healing after tooth extraction with ridge preservation using mineralized versus demineralized freeze-dried bone allograft. J Periodontol 83: 329-336

53. Fucini S, Quintero G, Gher M, Black B, Richardson A (1993) Small versus large particles of demineralized freeze-dried bone allografts in human intrabony periodontal defects. J Periodontol 64: 844-847

54. Chen C, Wang H, Smith F, Glickman G, Shyr Y, et al. (1995) Evaluation of a collagen membrane with and without bone grafts in treating periodontal intrabony defects. J Periodontol 66: 838-847

55. Piattelli A, Scarano A, Corigliano M, Piatelli M (1996) Comparison of bone regeneration with the use of mineralized and demineralized freezedried bone allografts: a histological and histochemical study in man. Biomaterials 17: 1127-1131

56. Duval B, Maynard J, Gunsolley J, Waldrop T (2000) Treatment of human mucogingival defects utilizing a bioabsorbable membrane with and without a demineralized freeze-dried bone allograft. J Periodontol 71: $1687-1692$

57. Scarano A, Degidi M, Lezzi G, Pecora G, Piatelli M, et al. (2006) Maxillary sinus augmentation with different biomaterials: a comparative histologic and histomorphometric study in man. Implant Dent 15: 197-207

58. Quattlebaum J, Mellonig J, Hensel N (1988) Antigenicity of freeze-dried cortical bone allograft in human periodontal osseous defects. J Periodontol 59: 394-397

59. Kassolis J, Rosen P, Reynolds M (2000) Alveolar ridge and sinus augmentation utilizing platelet-rich plasma in combination with freezedried bone allograft: case series. J Periodontol 71: 1654-1661

60. Schwartz S, Koch M, Deas D, Powell C (2006) Combined endodonticperiodontic treatment of a palatal groove: a case report. Journal Endod 32: $573-578$

61. West J, Oates $\mathrm{T}$ (2007) Identification of stability changes for immediately placed dental implants. Int J Oral Maxillofac Implants 22: 623-630

62. El-Halaby A, Becker J, Bissada N (2009) Autogenous bone grafting in a patient on long-term oral bisphosphonate therapy: case report. Int JPeriodontics Restorative Dent 29: 634-641

63. Beitlitum I, Artzi S, Nemcovsky C (2010) Clinical evaluation of particulate allogeneic with and without autogenous bone grafts and resorbable collagen membranes for bone augmentation of atrophic alveolar ridges. Clin Oral Implants Res 21: 1242-1250

64. Vidal R, Greenwell H, Hill M, Papageorgakopoulos G, Scheetz J (2010) Success rate of immediate implants placed and restored by novice operators. Implant Dent 19: 81-90

65. Yun J-H, Jun CM, Oh N-S (2011) Secondary closure of an extraction socket using the double-membrane guided bone regeneration technique with immediate implant placement. J Periodontal Implant Sci 41: 253-258

66. Eskow A, Mealey B (2013) Evaluation of healing following tooth extraction with ridge preservation using cortical versus cancellous freeze dried bone allograft. J Periodontol Published ahead of print

67. Spinato S, Galindo-Moreno P (2014) Evaluation of buccal plate after human bone allografting: clinical and СBCT outcomes of immediate anterior implants in eight consecutive cases. Int J Periodontics Restorative Dent 34: e58-66

68. Lyford R, Mills M, Knapp C, Scheyer E, Mellonig J (2003) Clinical evaluation of freeze-dried block allografts for aveolar ridge augmentation: a case series. Int J Periodontics Restorative Dent 23: $417-425$

69. Nissan J, Romanos G, Mardinger O, Chaushu G (2008) Immediate nonfunctional loading of single-tooth implants in the anterior maxilla following augmentation with freeze-dried cancellous block allograft: a case series. Int J Oral Maxillofac Implants 23: 709-716

70. Nissan J, Mardinger O, Calderon S, Romanos G, Chashu G (2011) Cancellous bone block allografts for the augmentation of the anterior atrophic maxilla. Clin Implant Dent Relat Res 13: 104-111 
Citation: Samsell B, Moore M, Bertasi G, Spinato S, Bernardello F, et al. (2014) Are Bone Allografts Safe and Effective for Today's Dental Practitioner?. Dentistry 4: 260. doi:10.4172/2157-7633.1000260

Page 6 of 6

71. Chaushu G, Mardinger O, Calderon S, Moses O, Nissan J (2009) The use of cancellous block allograft for sinus floor augmentation with simultaneous implant placement in the posterior atrophic maxilla. J Periodontol 80: 422-428

72. Wallace S, Gellin R (2008) Clinical evaluation of a cancellous block allograft for ridge augmentation and implant placement: a case report. Implant Dent 17: 151-158

73. Wallace S, Gellin R (2010) Clinical evaluation of a cancellous block allograft for ridge augmentation and implant placement in the maxilla Implant Dent 19: 272-276
74. Wallace S (2013) Guided bone regeneration for socket preservation in molar extraction sites: histomorphometric and 3D computerized tomography analysis. J Oral Implantol 39: 503-509

75. Bernardello F, Massaron E, Spinato S, Zaffe D (2014) Two-stage crestal sinus elevation by sequential drills in less than $4 \mathrm{~mm}$ of residual ridge height: a clinical and histologic case report. Implant Dent 23: 378-386 